\title{
Utilização do filtrado CCE na deslignificação com oxigênio e seus efeitos na branqueabilidade, refinabilidade e propriedades do papel
}

\author{
Effect of the CCE filtrated in the oxygen delignification on the \\ bleachability, refinability and properties of the paper
}

\author{
Janaína de Oliveira Resende' ${ }^{1}$, Jorge Luiz Colodette ${ }^{2}$, Marcela Freitas Andrade ${ }^{2}$
}

\begin{abstract}
RESUMO
A produção de polpa solúvel utilizando o estágio CCE (could caustic extraction = extração cáustica a frio), gera quantidades substanciais de licor CCE rico em hemiceluloses sendo importante o aproveitamento deste resíduo por razões econômicas e ambientais. Uma das potenciais aplicações das hemiceluloses recuperadas é como aditivo ao processo de fabricação de papéis. O objetivo deste estudo foi avaliar a redeposição de xilanas de licor CCE obtidas por precipitação dos sólidos do licor com etanol em polpa kraft não branqueada de eucalipto durante o processo de pré- $\mathrm{O}_{2}$, aliando ganhos de rendimento com melhoria de propriedades da polpa. As xilanas precipitadas foram aplicadas à polpa em quatro dosagens diferentes em relação a massa de polpa $(5,10,15$ e $20 \%)$. Os resultados foram interpretados com base no rendimento do processo, e no teor de xilanas, na branqueabilidade, na refinabilidade e nas propriedades físicas da polpa. As polpas que receberam adição de xilanas tiveram ganhos de rendimento na faixa de $-1,3$ a $4,5 \%$ em relação a polpa referência. A refinabilidade das polpas foi substancialmente melhorada pela adição de xilanas, sendo o consumo de energia reduzido em $70,3 \%$, para uma aplicação de $20 \%$ de xilanas, no grau de resistência à drenagem de $55^{\circ} \mathrm{SR}$. De modo geral, as propriedades de resistência dos papéis branqueados foram beneficiadas pela adição de xilanas.
\end{abstract}

Palavras-chave: polpa solúvel, hemiceluloses, redeposição de xilanas.

\begin{abstract}
The production of dissolving pulp thought the use of the CCE (Cold Caustic Extraction) technique generates a substantial volume of CCE liquor with large amount of hemicelluloses, and it is important the use this residue for economic and environmental reasons. One of the potential applications of the recovered hemicellulose is as additive in the paper manufacturing process. The objective of this study was to evaluate the deposition of xylan obtained from CCE liquor by liquor solids precipitation with ethanol in eucalyptus Kraft pulp not bleached during the pre- $\mathrm{O}_{2}$, combining efficiency gains with improved pulp properties. The precipitated xylan was applied to the pulp in four different percentages to the pulp mass $(5,10,15$ and $20 \%)$. The results were interpreted based on process yield, and xylan content, the bleachability, refinability and physical properties of the pulp. The pulps which received the addition of xylan had a production increase in the range of $-1,3$ to $4,5 \%$ compared to reference pulp. The refinability of the pulp was substantially improved by the addition of xylan, with reduced energy consumption by $70.3 \%$ to $20 \%$ xylan addition on the degree of resistance to drain by $55^{\circ} \mathrm{SR}$. Generally, the strength properties of bleached paper were benefited by the addition of xylan.
\end{abstract}

keywords: soluble pulp, hemicelluloses, xylan deposition.

\section{INTRODUÇÃO}

A indústria de celulose e papel vem se desenvolvendo nos últimos anos com intuito de estabelecer em suas indústrias o importante conceito de sustentabilidade, aproveitando ao máximo todos os constituintes da matéria prima utilizada, a madeira. Por isso, muitas pesquisas vêm sendo desenvolvida com objetivo de reduzir custos de produção e impacto ambiental, melhorando a qualidade dos produtos.

1. Klabin. Telêmaco Borba / PR, Brasil.

2. Universidade Federal de Viçosa, UFV. Viçosa / MG, Brasil. E-mail: andrade-marcela@hotmail.com

Sci. For., Piracicaba, v. 46, n. 120, p. 657-669, dez. 2018 DOI: dx.doi.org/10.18671/scifor.v46n120.14 
Polpas solúveis são polpas especiais que possuem alto teor de alfa-celulose, e baixos teores de hemiceluloses, lignina, extrativos e inorgânicos (SIXTA, 2006). Elas podem ser produzidas a partir de polpa kraft para papel, utilizando-se uma técnica de remoção de hemiceluloses denominada de extração alcalina a frio (CCE). Para viabilizar economicamente a etapa CCE, é necessária a recuperação do licor branco, para reuso na própria CCE e em outras etapas do processo produtivo (exemplo: cozimento e pré- $\mathrm{O}_{2}$ ) e recuperação das hemiceluloses para aplicações em fins nobres no processo de fabricação de papel (aditivos em papel P\&W) e em processos de biorrefinaria (bio-polímeros, adsorventes, biofilmes, etc.).

O uso de xilanas como aditivo ao processo de fabricação de polpa e papel pode modificar as propriedades do papel resultante de maneira positiva (BARBOSA et al., 2016; PEDRAZZI, 2009; SCHÖNBERG et al., 2001, SOARES, 2009). Além de aumentar o rendimento da linha de fibras, as hemiceluloses facilitam o refino da polpa e aumentam a quantidade de ligações entre fibras, resultando em ganhos de propriedades físicas e mecânicas do papel (PEDRAZZI, 2009).

A separação das hemiceluloses do licor branco no filtrado alcalino é uma operação complexa, para a qual não existe tecnologia disponível na atualidade. Nesse estudo, foi utilizada a técnica de precipitação do licor CCE com etanol, seguida de centrifugação para separação das hemiceluloses do licor branco.

O objetivo deste trabalho foi desenvolver um estudo para aproveitamento de xilanas, através da sua deposição à polpa kraft convencional de eucalipto na etapa de deslignificação com oxigênio, avaliando-se a influência delas nas etapas de deslignificação com oxigênio, branqueamento e refinação da polpa e, de suas consequências no rendimento e nas propriedades do papel.

\section{MATERIAL E MÉTODOS}

\section{Plano de Trabalho}

Uma polpa de eucalipto kraft pré- $\mathrm{O}_{2}$ foi tratada com licor branco e o licor CCE resultante foi recuperado. As xilanas presentes do licor CCE foram isoladas por precipitação com etanol, seguida de centrifugação. As xilanas isoladas foram depositadas sobre a polpa, na etapa de pré- $\mathrm{O}_{2}$, para produção de polpa P\&W. A Figura 1 mostra, esquematicamente, o plano de trabalho executado.

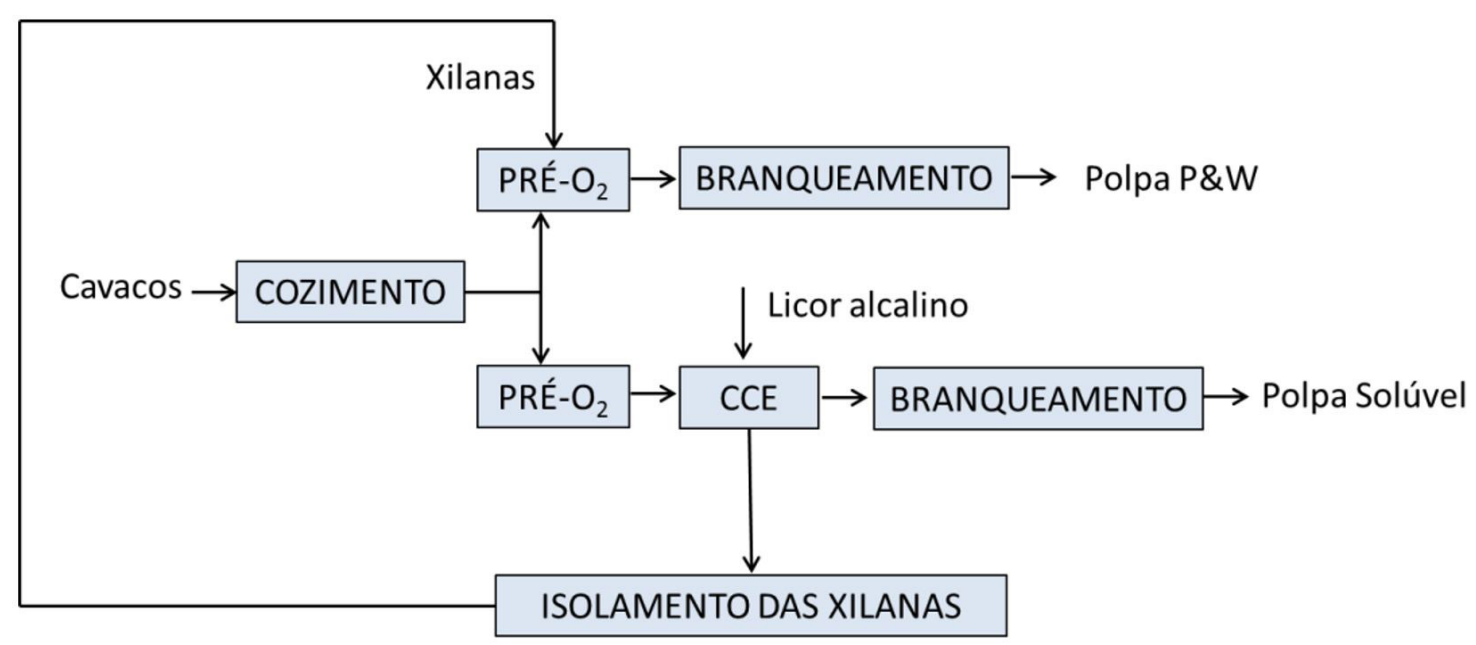

Figura 1. Plano de trabalho para deposição de xilanas

Figure 1. Working plan for xylans deposition

\section{Material}

As principais características das polpas pré- $\mathrm{O}_{2}$ e marrom de eucalipto utilizadas nesse estudo estão apresentadas na Tabela 1. 
Tabela 1. Características gerais das polpas pré- $\mathrm{O}_{2}$ e marrom

Table 1. General characteristics of pre- $\mathrm{O}_{2}$ and brown pulps

\begin{tabular}{|c|c|c|}
\hline Caracteristicas da polpa & Polpa Pré-O & Polpa marrom \\
\hline Número Kappa & 11,7 & 12,8 \\
\hline Alvura, \% ISO & 52,4 & 41,5 \\
\hline Viscosidade, $\mathrm{dm}^{3} / \mathrm{kg}$ & 919 & 935 \\
\hline $\mathrm{S} 10, \%$ & 17,4 & - \\
\hline $\mathrm{S} 18, \%$ & 13,5 & - \\
\hline a-celulose, $\%$ & 81,8 & - \\
\hline Pentosanas, $\%$ & 16,4 & - \\
\hline Cinzas (\%) & 2,2 & - \\
\hline Lignina, \% & - & 0,7 \\
\hline Lignina solúvel, \% & - & - \\
\hline HexA, \% & - & 0,8 \\
\hline
\end{tabular}

\section{Métodos}

\section{Produção do licor CCE}

O estágio CCE foi efetuado em sacos de polietileno, utilizando-se licor alcalino (licor branco, como álcali efetivo - AE). Amostras de $150 \mathrm{~g}$ a.s. de polpa foram tratadas com o equivalente a $550 \mathrm{~kg} \mathrm{AE}$ (como AE)/tas de polpa, por $15 \mathrm{~min}, 2^{\circ} \mathrm{C}$, na consistência de $15 \%$. O licor branco foi adicionado à polpa e após mistura manual e tempo de solubilização, o chamado licor CCE foi extraído da polpa por meio de centrifugação durante 30 minutos em sacos de tecido.

\section{Precipitação de xilanas do licor CCE}

As xilanas presentes no licor CCE foram precipitadas no meio utilizando etanol. Este processo foi realizado adicionando etanol 92\% em 5 litros de licor CCE na proporção de 1:1. A solução foi homogeneizada e em seguida deixada em repouso durante 24 horas para decantação do precipitado. A solução sobrenadante foi removida por meio de bomba de sucção a vácuo e o precipitado remanescente foi lavado com mais duas dosagens de etanol (3 litros em cada), sendo a sobrenadante removida novamente por bomba a vácuo.

Após as três sequências de lavagem o precipitado foi colocado em sacos de tecido e centrifugado. As xilanas precipitadas foram mantidas em ambiente a temperatura de $25^{\circ} \mathrm{C}$ para secagem. Após secarem parcialmente, as xilanas foram classificadas em peneiras de 25 mesh e colocadas em ambiente a temperatura de $25{ }^{\circ} \mathrm{C}$ para secagem final para determinação do teor seco (a.s).

\section{Deposição de xilanas na Pré $\mathbf{O}_{2}$}

As dosagens de xilanas precipitadas foram dissolvidas na solução de $\mathrm{NaOH}$ que foi utilizada durante a pré- $\mathrm{O}_{2}$. Como referência, foi realizado um estágio de pré- $\mathrm{O}_{2^{\prime}}$ sem adição de xilanas. A pré- $\mathrm{O}_{2}$ foi efetuada em um reator/misturador modelo Mark V (Quantum Technologies Inc.) com amostras de $250 \mathrm{~g}$ de polpa a.s. nas condições apresentadas na Tabela 2. As dosagens de xilanas foram $0,5,10,15$ e 20\% em relação à massa de polpa, de modo que as polpas foram nomeadas após o procedimento como Referência (sem adição de xilana), X5 (5\% de xilana), X10 (10\% de xilana), X15 (15\% de xilana), X20 (20\% de xilana).

Tabela 2. Condições do branqueamento das polpas referência, $X 5, X 10, X 15$ e X20.

Table 2. Bleaching conditions of the reference pulps, $X 5, X 10, X 15$ and $X 20$.

\begin{tabular}{lccccc}
\multicolumn{1}{c}{ Condições e Resultados } & Pré- $_{2}$ & Do & (EP) & D & P \\
\hline Consistência, $\%$ & 10 & 11 & 11 & 11 & 10 \\
Tempo, min & 75 & 120 & 90 & 90 & 60 \\
Temperatura, ${ }^{\circ} \mathrm{C}$ & 105 & 95 & 80 & 75 & 80 \\
$\mathrm{H}_{2} \mathrm{O}_{2}, \mathrm{~kg} / \mathrm{t}$ & - & - & 4 & - & Variável \\
$\mathrm{ClO}_{2}, \mathrm{Kg} / \mathrm{t}$ & - & $\mathrm{FK}=0,23$ & - & 10 & - \\
\hline
\end{tabular}


Resende, Colodette e Andrade. - Utilização do filtrado CCE na deslignificação com oxigênio e seus efeitos na branqueabilidade, refinabilidade e

propriedades do papel

\section{Branqueamento ECF para produção de polpa P\&W}

As condições de todas as etapas de branqueamento com a sequência OD(EP)DP estão apresentadas na Tabela 2. A dosagem de reagentes variou de acordo com o comportamento de cada polpa em relação à alvura.

\section{Refino e testes físicos e mecânicos}

As polpas branqueadas foram encaminhadas para refino para comprovação da sua qualidade como polpa de mercado por meio das análises físicas e mecânicas das polpas refinadas. As cinco diferentes polpas produzidas foram refinadas em moinho PFI em quatro níveis de refino. Esses níveis foram determinados em função de se alcançar o grau Shopper Rigler - ${ }^{\circ}$ SR definido (55), sendo que o nível 0 (sem refino) de refino foi realizado em todas as polpas, conforme tabela 3.

Tabela 3. Procedimentos analíticos dos testes físicos e mecânicos

Table 3. Analytical procedures of physical and mechanical tests

\begin{tabular}{lr}
\multicolumn{1}{c}{ Refinamento da polpa } & TAPPI248 sp-08 \\
\hline Formação de folhinhas para os testes físicos das polpas & TAPPI205 om-81 \\
Volume específico aparente (VEA) & TAPPI220 sp-01 \\
Peso específico aparente (PEA) & TAPPI 220 sp-01 \\
Gramatura do papel & TAPPI410 om-08 \\
Índice de Rasgo do papel & TAPPI414 om-98 \\
Índice de Tração do papel & TAPPI494 om-01 \\
Opacidade & TAPPI519 om-06 \\
Coeficiente de dispersão de luz & TAPPI1214 sp-98 \\
Teor de xilanas, \% & Wallis et al (1996) \\
Viscosidade, (dm3/kg) & TAPPI T230 om 99 \\
Número Kappa & TAPPI T236 om 99 \\
Alvura, \%ISO & TAPPI T525 om 92 \\
Teor de HexA, \% & TAPPI T 282 pm-07 \\
Teor de Lignina, \% & KlasonTAPPI T222 om-98 \\
Teor de Lignina Solúvel,\% & TAPPIUsefulmethod UM 250 \\
Reversão de alvura, \%ISO & TAPPI UM200 4h, 105 ${ }^{\circ} \mathrm{C}, 0 \%$ UR, após \\
& acondicionamento das folhas por $4 \mathrm{~h}$ em sala \\
\hline
\end{tabular}

\section{Análises estatísticas}

Para análise dos testes físico-mecânicos das polpas referência e com adição de xilanas foi utilizado o software Curve Expert 1.4 para obtenção dos modelos. As equações ajustadas foram comparadas pelo teste F, utilizando-se o teste de identidade de modelos e adotando-se um nível de significância de $5 \%$ de probabilidade, conforme metodologia apresentada por Regazzi (1993) para modelos lineares.

\section{RESULTADOS E DISCUSSÃO}

\section{Caracterização qualitativa e quantitativa das xilanas provenientes do licor CCE}

No processo de extração das xilanas da polpa houve remoção de 19,8\% de material (29,7 gramas de massa em $150 \mathrm{~g}$ de polpa seca); essa massa extraída estava presente num volume de $850 \mathrm{~mL}$, que é o volume equivalente a $150 \mathrm{~g}$ de polpa a.s numa consistência de 15\%. As xilanas são insolúveis em etanol e desta forma de precipitam na presença desse solvente, decantando após algumas horas em repouso. No processo de precipitação, algumas impurezas aparecem como contaminantes das xilanas. Foi verificada a composição da xilana precipitada. Após a precipitação com etanol, a massa de material contido no volume $850 \mathrm{~mL}$ cresceu de $29,7 \mathrm{~g}$ para 34,7g. Esse aumento é explicado pelo teor de cinzas derivado do hidróxido de sódio usado no estágio CCE, que foi parcialmente carreado junto com o precipitado de xilanas mesmo após a lavagem com etanol. Em uma massa de 34,7 g de material precipitado continha $37 \%$ de cinzas, $51,8 \%$ de xilanas, 2,8\% de lignina Klason, 0,3\% de lignina solúvel e $1,1 \%$ de hexa.

O grande inconveniente das hemiceluloses recuperadas pela técnica de precipitação/centrifugação é o seu elevado teor de cinzas. Como as cinzas são grandemente compostas de sódio, as hemiceluloses 
apresentam alto grau de alcalinidade e, idealmente, devem ser usadas como aditivos em ambiente que requer alta alcalinidade. Numa fábrica de polpa kraft, elas podem, potencialmente, serem aplicadas no cozimento ou na deslignificação com oxigênio (SOARES; COLODETTE, PEDRAZZI, 2010). Considerando-se que o material recuperado também contém lignina que pode ser facilmente removida nas operações de cozimento e de deslignificação com oxigênio.

A remoção de xilanas de polpa pré- $\mathrm{O}_{2^{\prime}}$ em vez de uma polpa branca, apresenta a desvantagem da maior presença de lignina e ácidos urônicos (especialmente HexAs), que estão ligados quimicamente às xilanas e são arrastados com elas no tratamento CCE. Porém, considerando o aproveitamento de xilanas geradas da produção de polpa solúvel, o estágio CCE realizado no início do processo na polpa não branqueada, resulta em benefícios de reduzir o consumo de reagentes de branqueamento e ser mais eficiente na remoção de xilanas.

\section{Efeito da adição de xilanas no desempenho da pré $\mathbf{O}_{2}$}

Xilanas extraídas e precipitadas do licor CCE foram redepositadas na etapa de deslignificação com oxigênio (pré- $\mathrm{O}_{2}$ ) de uma polpa marrom, visando ganhar rendimento e melhorar a refinabilidade e propriedades da polpa. Após a pré- $\mathrm{O}_{2}$ a polpa foi caracterizada quanto aos teores de xilanas, alvura, número kappa, HexA, lignina, rendimento e viscosidade como mostrado nas Figuras 2-5.

\section{Xilana}

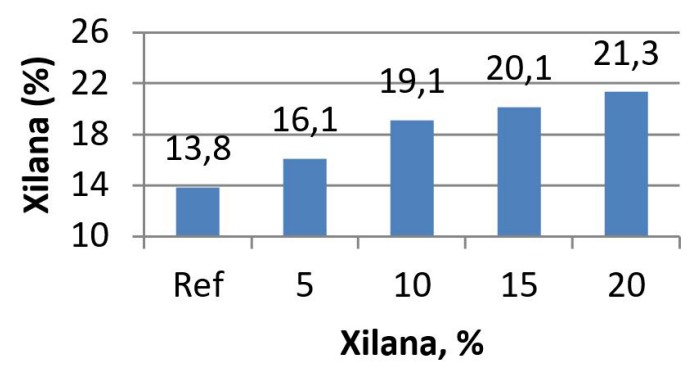

Figura 2. Teor de xilanas nas polpas Referência, $X 5, X 10, X 15$ e X20 após pré- $\mathrm{O}_{2}$ Figure 2. Content of xylans in reference pulps, X5, X10, X15 and X20 after pre- $\mathrm{O}_{2}$

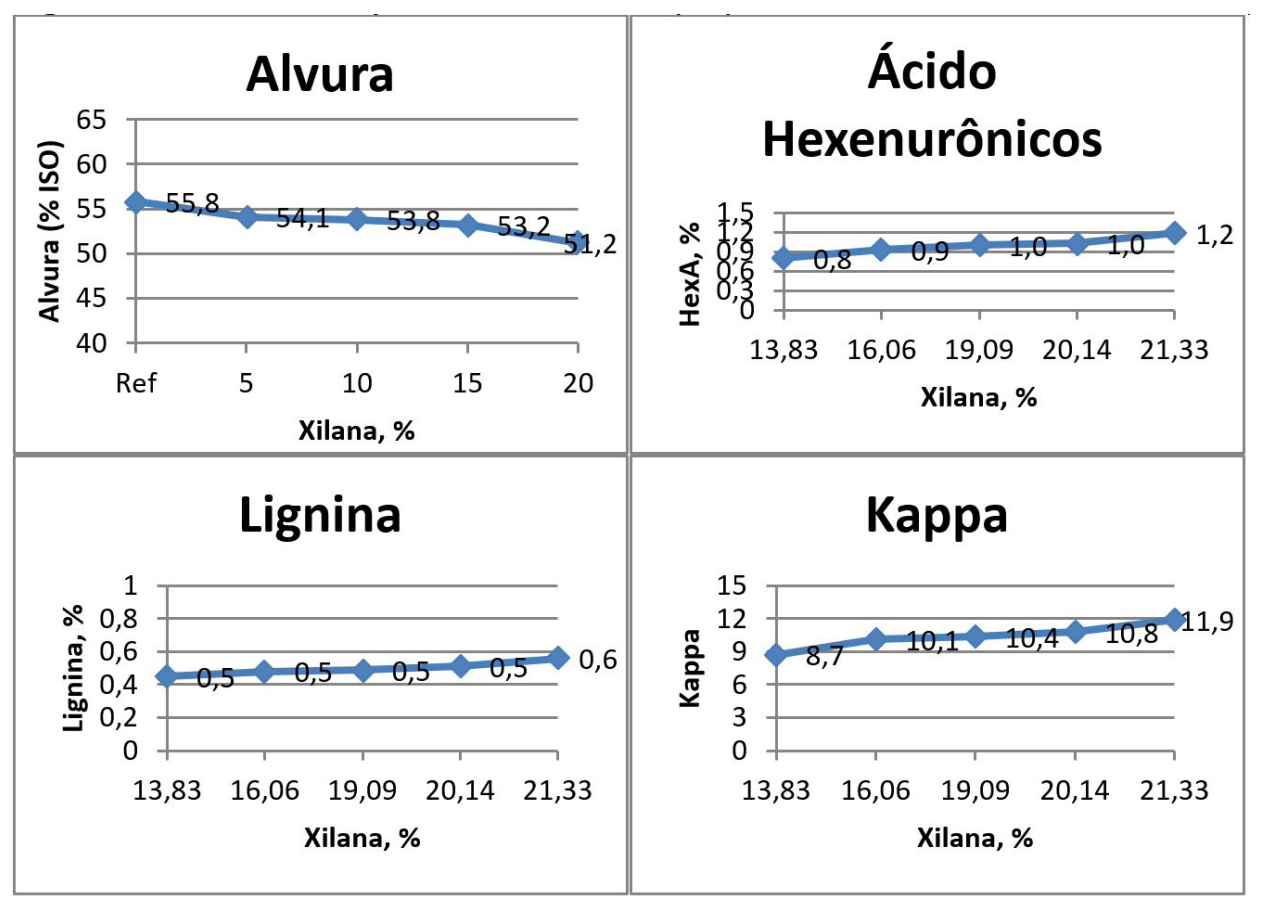

Figura 3. Alvura (A), Teor de HexA (B), lignina (C) e número kappa (D) das polpas Referência, X5, X10, X15 e X20 após pré- $\mathrm{O}_{2}$

Figure 3. Brightness $(A)$ hexa content $(B)$, lignin $(C)$ and kappa number $(D)$ of the reference pulps, $X 5, X 10, X 15$ and $\mathrm{X} 20$ after pre- $\mathrm{O}_{2}$ 
Resende, Colodette e Andrade. - Utilização do filtrado CCE na deslignificação com oxigênio e seus efeitos na branqueabilidade, refinabilidade e

propriedades do papel

\section{Viscosidade}

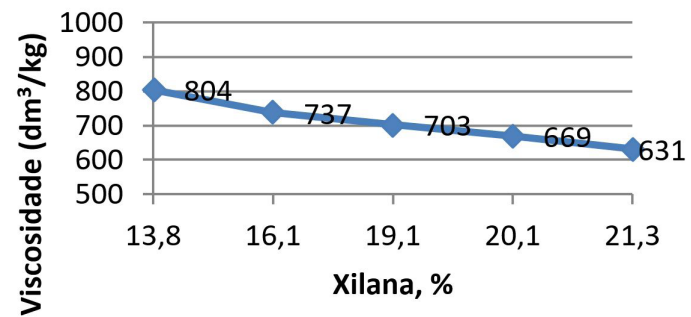

Figura 4. Viscosidade das polpas Referência, $X 5, X 10, X 15$ e X20 após pré- $\mathrm{O}_{2}$

Figure 4. Viscosity of reference pulps, $X 5, X 10, X 15$ and $X 20$ after pre- $\mathrm{O}_{2}$

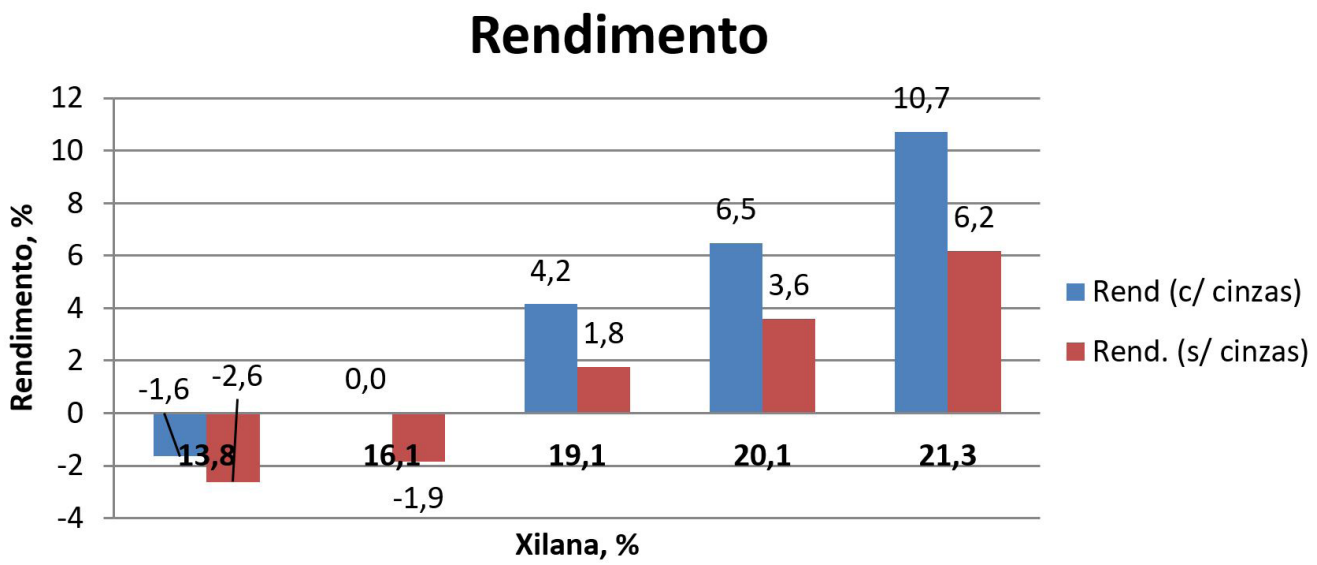

Figura 5. Rendimento das polpas Referência, X5, X10, X15 e X20 após pré- $\mathrm{O}_{2}$

Figure 5. Yield of the reference pulps, $\mathrm{X} 5, \mathrm{X} 10, \mathrm{X} 15$ and $\mathrm{X} 20$ after $\mathrm{pre}-\mathrm{O}_{2}$

Os resultados indicam que as dosagens de xilanas adicionadas na faixa de 5-20\% tem efeito significativo. A polpa marrom original continha $14,7 \%$ xilanas e após os procedimentos de deposição na pré- $\mathrm{O}_{2}$ o conteúdo de xilanas na polpa aumentou para um intervalo de $16,0-21,3 \%$, enquanto que na referência, a polpa que não recebeu xilanas, houve queda no teor de xilanas para 13,9\% devida a degradação destas (Figura 2).

As maiores dosagens de xilanas aplicadas geraram polpas com maiores valores de número kappa, HexA, lignina solúvel e menores valores de alvura (Figura 3). As xilanas aplicadas continham elevados valores de HexA e uma certa quantidade de lignina; isto porque elas foram obtidas de licor CCE proveniente de polpa pré- $\mathrm{O}_{2}$. Em se tratando deste tipo de polpa, esses componentes são solubilizados juntamente com as xilanas no processo alcalino de extração. Durante a precipitação com etanol estes compostos também são arrastados com a xilanas. Na pré- $\mathrm{O}_{2^{\prime}}$ esses compostos responsáveis pelo número kappa da polpa também se depositam nas fibras.

$\mathrm{O} \mathrm{pH}$ final das amostras foram controlados de forma a terminarem entre 11,5-12. Isto porque grande parte da lignina residual presente na polpa marrom possui estruturas que contem grupos hidroxílicos fenólicos livres, e caso o $\mathrm{pH}$ da pré- $\mathrm{O}_{2}$ terminar abaixo de 11, poderá ocorrer a precipitação da lignina sobre as fibras, diminuindo a qualidade da polpa. Por outro lado, a maior precipitação de xilanas nas fibras de celulose também ocorrem em pH abaixo de 12-12,5 (YLLNER; ENSTROM, 1957). Assim, é importante que o processo termine com quantidade adequada de íons $\mathrm{OH}^{-} \mathrm{de}$ tal forma a evitar a precipitação de lignina e maximizar a precipitação de xilanas (DANIELSSON, 2007).

Em relação a viscosidade foi possível observar que o aumento do teor de xilanas na polpa levou a redução da viscosidade (Figura 4), pois as xilanas são polímeros com peso molecular inferior ao da celulose. Desta forma, a proporção de cadeias menores aumenta na polpa e reduz a viscosidade.

A deposição de xilanas durante a deslignificação com oxigênio acarretou ganhos de rendimento (Figura 5). A etapa de deslignificação de polpas com oxigênio causa ligeira queda no rendimento devido à solubilização de lignina e de carboidratos de baixo peso molecular, especialmente xilanas.

No entanto, quando as xilanas foram depositadas durante a deslignificação com oxigênio, ocorreram ganhos no rendimento da pré- $\mathrm{O}_{2}$ de até $6,2 \%$, já descontada a perda de rendimento da polpa referência. 
Todavia, é importante ressaltar que ocorre também redeposição de uma grande quantidade de cinzas na polpa. Durante o processo de extração/precipitação de xilanas grande quantidade de hidróxido de sódio (cinzas) fica retido junto ao precipitado mesmo após lavagem. Essas cinzas que se redepositam na polpa durante a pré- $\mathrm{O}_{2}$ podem ser consideradas contaminantes e mascaram os resultados de rendimento, uma vez que este material é removido durante as etapas ácidas de branqueamento subsequentes. Na Tabela 4 são apresentados os reais ganhos de rendimento advindos da deposição de xilanas, descontados os valores de cinzas. Nota-se que os ganhos de rendimento caíram da faixa de $-0,58$ a $6,2 \%$ para a faixa de $-1,3$ a $4,5 \%$ sendo os ganhos ainda relevantes para as dosagens mais altas de xilanas. Observa-se ainda na Tabela 5 que o teor de lignina da polpa tendeu a crescer com a dosagem de xilanas, mas o teor de glicanas não foi grandemente alterado.

Tabela 4. Rendimento e teores de cinzas, lignina e glicanas das polpas Referência, $X 5, X 10, X 15$ e $X 20$ após pré- ${ }_{2}$ Table 4. Yields and ash, lignin and glycan content of reference pulps, X5, X10, X15 and X20 after pre- $\mathrm{O}_{2}$

\begin{tabular}{|c|c|c|c|c|c|c|c|c|}
\hline Amostra & $\begin{array}{c}\text { Rendimento } \\
(\%)\end{array}$ & $\begin{array}{c}\text { Rendimento } \\
\text { sem } \\
\text { cinzas* } \\
(\%)\end{array}$ & $\begin{array}{l}\text { Massa } \\
\text { Pós } \\
\text { Pré-O } \\
\text { (g) }\end{array}$ & $\begin{array}{c}\text { Cinzas } \\
(\%)\end{array}$ & $\begin{array}{l}\text { Lignina } \\
\qquad \%)\end{array}$ & $\begin{array}{c}\text { Glicanas } \\
(\%)\end{array}$ & $\begin{array}{l}\text { Massa de } \\
\text { Glicanas } \\
\text { (g) }\end{array}$ & $\begin{array}{c}\text { Xilanas } \\
\text { não } \\
\text { retidas (\%) }\end{array}$ \\
\hline MARROM & - & - & 250 & - & 0,7 & 79,6 & 199 & - \\
\hline REFERÊNCIA & $-1,64$ & $-2,6236$ & 245,9 & 1 & 0,5 & 79,2 & 194,8 & - \\
\hline$\times 5$ & 0,04 & $-1,8608$ & 250,1 & 1,9 & 0,5 & 77,4 & 193,6 & 50,7 \\
\hline$\times 10$ & 4,16 & 1,76432 & 260,4 & 2,3 & 0,5 & 73,3 & 190,9 & 37,2 \\
\hline X15 & 6,48 & 3,60504 & 266,2 & 2,7 & 0,5 & 72,9 & 194,1 & 47,7 \\
\hline $\mathrm{X} 20$ & 10,72 & 6,18048 & 276,8 & 4,1 & 0,6 & 70,2 & 194,3 & 49,9 \\
\hline
\end{tabular}

Tabela 5. Resumo dos resultados do branqueamento para as polpas Referência, X5, X10, X15 e X20.

Table 5. Summary of bleaching results for Reference pulps, X5, X10, X15 and X20.

\begin{tabular}{|c|c|c|c|c|c|}
\hline & Referência & $\mathbf{X 5}$ & $\mathrm{X} 10$ & $\mathbf{X 1 5}$ & $\mathbf{X} 20$ \\
\hline Número kappa da polpa antes branqueamento (polpa Pré- $\mathrm{O}_{2}$ ) & 8,7 & 10,1 & 10,4 & 10,8 & 11,9 \\
\hline Viscosidade da polpa antes branqueamento (polpa Pré- $\mathrm{O}_{2}$ ) & 804 & 737 & 703 & 669 & 631 \\
\hline Xilanas da polpa antes branqueamento (polpa pré- $\mathrm{O}_{2}$ ) & 13,9 & 16,1 & 19,1 & 20,1 & 21,3 \\
\hline $\mathrm{ClO}_{2}, \mathrm{~kg} / \mathrm{tas}$ como $\mathrm{Cl}_{2}$ & 46,4 & 49,5 & 50,1 & 51,1 & 53,6 \\
\hline $\mathrm{H}_{2} \mathrm{SO}_{4} \mathrm{~kg} / \mathrm{tas}$ & 3,7 & 6,5 & 9,4 & 10,6 & 18 \\
\hline $\mathrm{NaOH}, \mathrm{kg} / \mathrm{tas}$ & 12,0 & 13,6 & 15,5 & 15,5 & 15,9 \\
\hline $\mathrm{H}_{2} \mathrm{O}_{2}, \mathrm{~kg} / \mathrm{tas}$ & 6,0 & 6,3 & 7,9 & 7,5 & 10,3 \\
\hline $\mathrm{CAT}^{1}, \mathrm{~kg} / \mathrm{tas}$ & 58,9 & 62,7 & 66,6 & 66,8 & 75,1 \\
\hline Viscosidade, $\mathrm{dm}^{3} / \mathrm{kg}$ & 665 & 626 & 590 & 599 & 560 \\
\hline Alvura, \% ISO (interpolado) & 90 & 90 & 90 & 90 & 90 \\
\hline Reversão de Alvura, \% ISO & 2,9 & 3,3 & 3,3 & 3,5 & 3,7 \\
\hline HexA, \% & 0,1 & 0,1 & 0,2 & 0,2 & 0,2 \\
\hline Xilanas polpa branqueada, \% & 13,9 & 15,5 & 18,5 & 19,7 & 20,6 \\
\hline Perda de xilanas no branqueamento ${ }^{2}, \%$ & 0 & 0,6 & 0,6 & 0,4 & 0,7 \\
\hline Perda de viscosidade no branqueamento ${ }^{3}, \mathrm{dm}^{3} / \mathrm{kg}$ & 139 & 111 & 113 & 70 & 71 \\
\hline
\end{tabular}

${ }^{1}$ Total Active Chlorine $=\left(\mathrm{ClO}_{2}+\mathrm{H}_{2} \mathrm{O}_{2} * 2,09\right) ;{ }^{2}$ perda de xilana $=$ Xilanas da polpa antes branqueamento - xilanas no branqueamento; ${ }^{2}$ perda de viscosidade $=$ Viscosidade da polpa antes branqueamento - viscosidade no branqueamento

\section{Efeito da adição de xilanas na pré- $\mathrm{O}_{2}$ na branqueabilidade da polpa}

Todas as polpas foram branqueadas para obter uma alvura final de 90\%ISO com a sequência D(EP)DP. Um resumo da quantidade de reagentes necessário para atingir esse teto de alvura está apresentado na Tabela 5.

As polpas tratadas com maiores quantidades de xilanas consumiram mais reagentes, expresso como cloro ativo total, para atingir em alvura de $90 \%$ ISO, em relação à referência. O aumento no consumo se deve ao aumento do número kappa após a pré- $\mathrm{O}_{2}$. Esse resultado era esperado, pois as xilanas precipitadas continham certa quantidade de lignina, que depositaram na polpa durante o estágio de deslignificação com oxigênio a um ponto que o número kappa pós-deslignificação com oxigênio foi maior para as polpas tratadas com xilanas do que para a referência. O aumento no consumo total de cloro ativo, com o aumento da aplicação de xilanas, ocorreu no estágio de deslignificação com dióxido de cloro e no estágio final de peroxidação. No primeiro caso, foi mantido um fator kappa 
Resende, Colodette e Andrade. - Utilização do filtrado CCE na deslignificação com oxigênio e seus efeitos na branqueabilidade, refinabilidade e

propriedades do papel

constante de 0,23, sendo que a dose de dióxido aumentou em razão da elevação do número kappa. Também foi necessário aumentar a dose de peróxido no estágio P para alcançar 90\% ISO de alvura.

Durante o branqueamento, o teor de xilanas nas polpas permaneceu estável, perdendo menos de $1 \%$ das xilanas depositadas. Este resultado é explicado pelo fato da estrutura das xilanas terem grande afinidade com as fibrilas de celulose. Soares (2009) argumenta que as xilanas extraídas de polpas brancas apresentam melhor afinidade com a polpa que as xilanas extraídas de polpa marrom, apresentando maior estabilidade durante o branqueamento. Ainda de acordo Soares (2009), as xilanas de polpa marrom apresentam grupos de ácidos urônicos que tornam a cadeia menos linear prejudicando a precipitação sobre a fibras. Os resultados desse estudo mostraram boa estabilidade para as xilanas depositadas, provenientes de polpa pré- $\mathrm{O}_{2^{\prime}}$ apesar de estas conterem altos teores de HexA. A adsorção das xilanas também afetou a reversão de alvura, que apresentou valores mais elevados para polpas com redeposição de xilanas. Maiores redeposições acarretam em maiores teores de HexA nas polpas, que pode causar os níveis de reversão de alvura mais altos.

Durante o branqueamento, a queda de viscosidade das polpas com a adição de xilanas foi menor que a referência (Tabela 5). Segundo Pedrazzi (2009), as xilanas desempenham o papel de proteção das cadeias de celulose contra a degradação dos reagentes.

\section{Efeito da adição de xilanas na pré-O ${ }_{2}$ na refinabilidade da polpa}

O refino é uma das principais etapas na produção da indústria papeleira. Uma boa refinabilidade da polpa é essencial para o alcance de excelência do seu produto.

Um importante parâmetro que foi avaliado é a estabilidade das xilanas após o processo de refino, pois caso as mesmas apresentem um decréscimo em seu teor, podem inviabilizar todo o processo devido às perdas de rendimento conquistadas pela adição da mesma. Por meio de análises dos resultados da composição química das polpas refinadas, foi observado que para todos os níveis de refino o teor de xilanas nas polpas permaneceu estável, perdendo menos de $1 \%$ de xilanas em média nas polpas X5, X10, X15 e X20 (Figura 6).

As polpas celulósicas contendo maiores teores de hemiceluloses são aptas a produzir papéis com melhores ligações entre fibras dando assim origem a papéis com maiores resistências mecânicas, melhor lisura superficial, mas menor volume específico aparente e porosidade (PEDRAZZI, 2009).

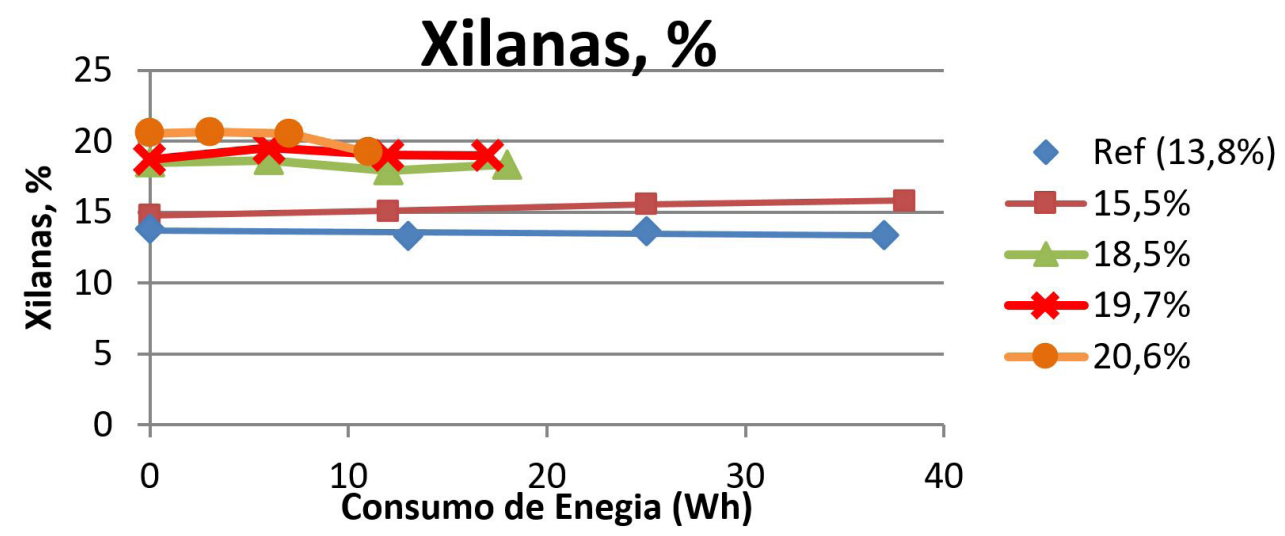

Figura 6. Comportamento do teor de xilanas das polpas referência, X5, X10, X15 e X20 após refino. Figure 6. Behavior of xylans content of reference pulps, X5, X10, X15 and X20 after refining.

Porém, elevado teor de xilanas também podem trazer pontos negativos na produção de papel, como por exemplo, no processo de drenagem que pode ser prejudicado, uma vez que as hemiceluloses são altamente hidrofílicas. Desta forma, o teor de xilanas deve ser controlado de acordo com as necessidades e condições de produção requeridas do papel.

O parâmetro refinabilidade é considerado neste trabalho como a quantidade de energia (W.h) necessária para se alcançar um determinado grau de resistência da polpa à drenagem (ex: ${ }^{\circ}$ Schopper Riegler - ${ }^{\circ} \mathrm{SR}$ ). O refino é um processo que consome grande quantidade de energia, sendo um parâmetro importante na composição do custo de fabricação de papéis que necessitam ser refinados. Portanto, polpas de boa refinabilidade são altamente desejáveis para o fabricante de papel. A análise dos dados 
de consumo de energia consumida no refino versus o ${ }^{\circ} \mathrm{SR}$ das polpas (Figura 7) mostrou tendências diferentes entre a referência e as polpas ricas em xilanas. Houve clara tendência de melhoria na refinabilidade pela adição de xilanas. A Figura 7 mostra que o mesmo ${ }^{\circ}$ SR foi alcançado pelas polpas com adição de xilanas com menos energia do que a exigida pela referência. Estes resultados estão coerentes com o observado na literatura (ANJOS et al., 2005).

Paralelamente ao ${ }^{\circ}$ Schopper Riegler, a drenagem na máquina de papel pode ser negativamente afetada, pois polpas ricas em hemiceluloses apresentam maior habilidade em atrair e em reter água na parede celular, uma vez que elas são altamente hidrofílicas.

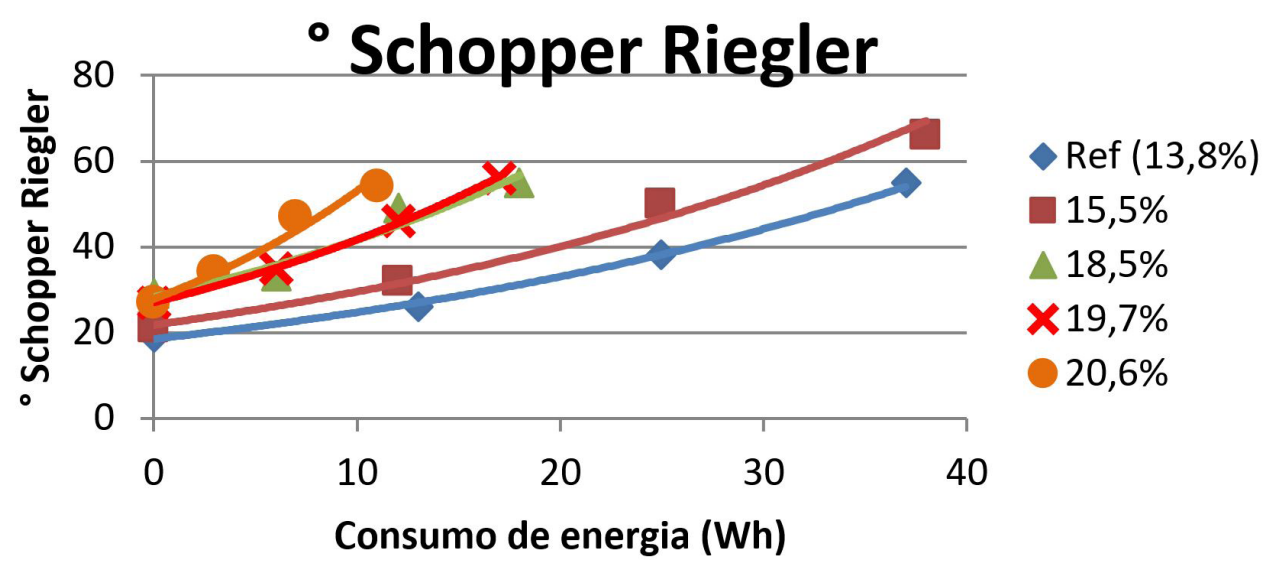

Figura 7. Variação do ${ }^{S}$ Schopper Riegler das polpas Referência, X5, X10, X15 e X20 em função do consumo de energia após cada nível de refino.

Figure 7. Variation of oSchopper Riegler of reference pulps, $X 5, X 10, X 15$ and $X 20$ in terms of the energy consumption after each refining level.

\section{Efeito da adição de xilanas na pré- $\mathrm{O}_{2}$ nas propriedades estruturais mecânicas e óticas da polpa}

As polpas branqueadas contendo diferentes quantidades de xilanas foram refinadas em moinho laboratorial PFI e avaliadas quanto às suas propriedades físicas, com o objetivo de caracterizar as polpas para produção de papéis P\&W. Para facilitar comparações de propriedades entre tratamentos, foram produzidas curvas de desenvolvimento de propriedades em relação ao consumo de energia no refino.

A resistência à tração é um dos mais importantes atributos de qualidade dos papéis $\mathrm{P} \& \mathrm{~W}$, sendo esta propriedade melhorada com o processo de refinação. As madeiras que possuem fibras com paredes mais delgadas são mais facilmente refinadas, fibrilam e colapsam mais facilmente, dando origem a folhas densas e resistentes (MOKFIENSKI et al., 2008). Outro fator que afeta a fibrilação e colapsamento é o conteúdo de hemiceluloses da fibra.

A Figura 8 apresenta curvas de índice de tração para os papéis produzidos em função do consumo de energia de refino das 5 polpas produzidas (Referência, X5, X10, X15 e X20). É possível observar que os maiores valores de tração são encontrados para as polpas com maiores níveis de xilanas.

As hemiceluloses contribuem fortemente para as ligações intra e interfibras, resultando em papéis refinados mais resistentes à tração (MILANEZ et al., 1982), o que pode ser visto claramente neste estudo. Além disso, o consumo de energia para o refino das polpas com elevado conteúdo de xilanas foi bastante inferior ao das demais polpas, mostrando que polpas com $15 \%$ e $20 \%$ de xilanas (X15 e X20) alcançam valores desejados de refino com considerável economia de energia para o processo. Resultados semelhantes foram encontrados por Pedrazzi (2009), onde polpas com mais de $20 \%$ de xilanas apresentaram maiores índice de tração, reduzindo o consumo de energia no refino dessas polpas.

Com base no teste F, a hipótese de igualdade das equações Referência e X5 não foram rejeitadas, podendo-se concluir que as duas amostras não diferem significativamente $(\mathrm{P}>0,05)$. Assim, uma curva comum pode ser usada como estimativa para as amostras iguais. Como o modelo estatístico 
Resende, Colodette e Andrade. - Utilização do filtrado CCE na deslignificação com oxigênio e seus efeitos na branqueabilidade, refinabilidade e

propriedades do papel

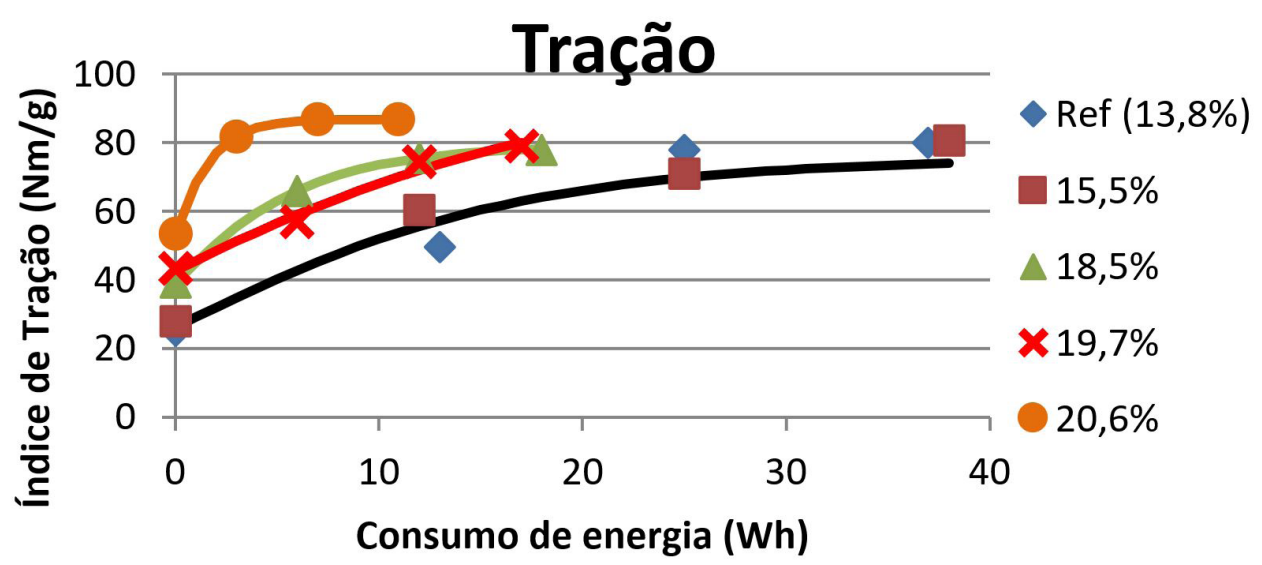

Figura 8. Índice de tração das polpas Referência, X5, X10, X15 e X20 em função do consumo de energia após cada nível de refino.

Figure 8. Tensile index of Reference pulps, $X 5, X 10, X 15$ and $X 20$ in terms of the energy consumption after each refining level.

não é o mesmo para as outras condições (X10, X15 e X20), pode-se concluir que existem diferenças entre as amostras.

$\mathrm{O}$ índice de rasgo está intimamente relacionado com a integridade da parede celular da fibra, número de fibras, ligações internas das fibras (CASTANHO; OLIVEIRA, 2000). A Figura 9 mostra que as polpas tratadas apresentaram valores de índice de rasgo maior que o da referência para menores níveis de refino. Porém, em maiores níveis de refino observou-se uma queda de do índice de rasgo nas polpas com maiores teores de xilanas. Salomão (2001) observou a mesma tendência em polpas refinadas de eucaliptos e afirmou que as hemiceluloses melhoram a ação do refino, mas, ao mesmo tempo, diminuem a relação índice tração versus índice de rasgo devido à diminuição no conteúdo proporcional de celulose. Além disso, refinamento da polpa causa a redução do comprimento médio das fibras, devido à ação corte das lâminas do refinador.

Com base no teste F, a hipótese de igualdade das amostras X15 e X20 não foi rejeitada, podendo-se concluir que as duas equações não diferem significativamente $(\mathrm{P}>0,05)$. Assim, uma curva comum

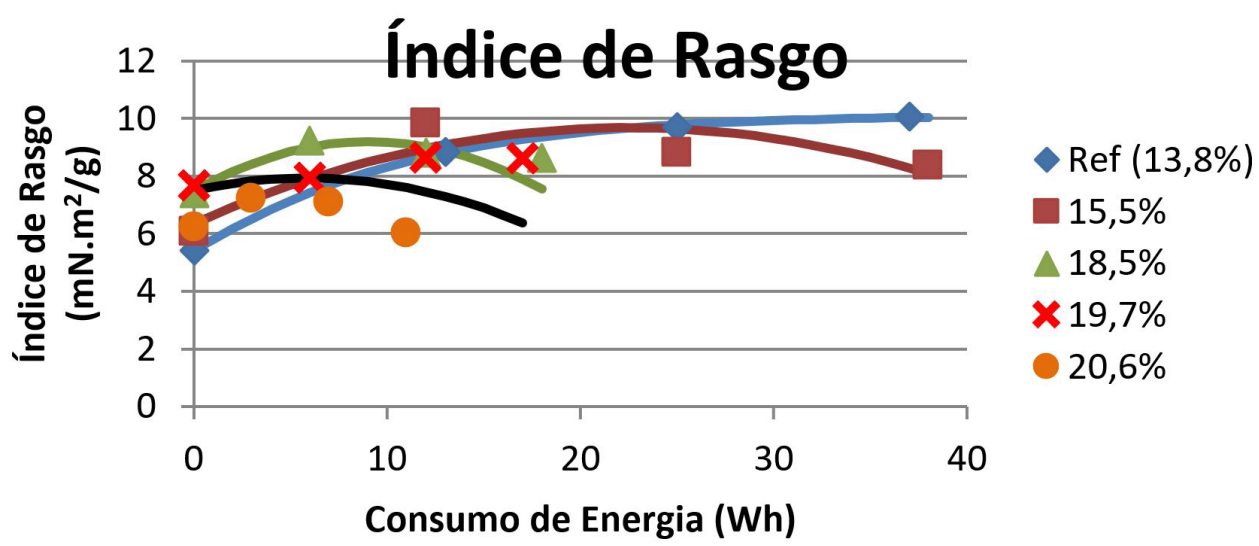

Figura 9. Índice de rasgo das polpas Referência, X5, X10, X15 e X20 em função do consumo de energia após cada nível de refino.

Figure 9. Tear Index of Reference pulps, X5, X10, X15 and X20 in terms of the energy consumption after each level of refining.

pode ser usada como estimativa para as amostras iguais, enquanto para as demais polpas o modelo estatístico não é o mesmo, de forma que se pode concluir que existem diferenças para estes experimentos.

O módulo de elasticidade específico (MOE) indica a rigidez do papel. A Figura 10 mostra que as polpas contendo xilanas depositadas melhoraram os valores de $\mathrm{MOE}$ em relação à referência. $\mathrm{O}$ valor do MOE é aumentado na medida em que se aumenta a energia consumida durante o refino.

Com base no teste F, a hipótese de igualdade das amostras Referência, X5 e X10 não foi rejeitada, podendo-se concluir que as três amostras não diferem significativamente $(\mathrm{P}>0,05)$. Assim, uma 


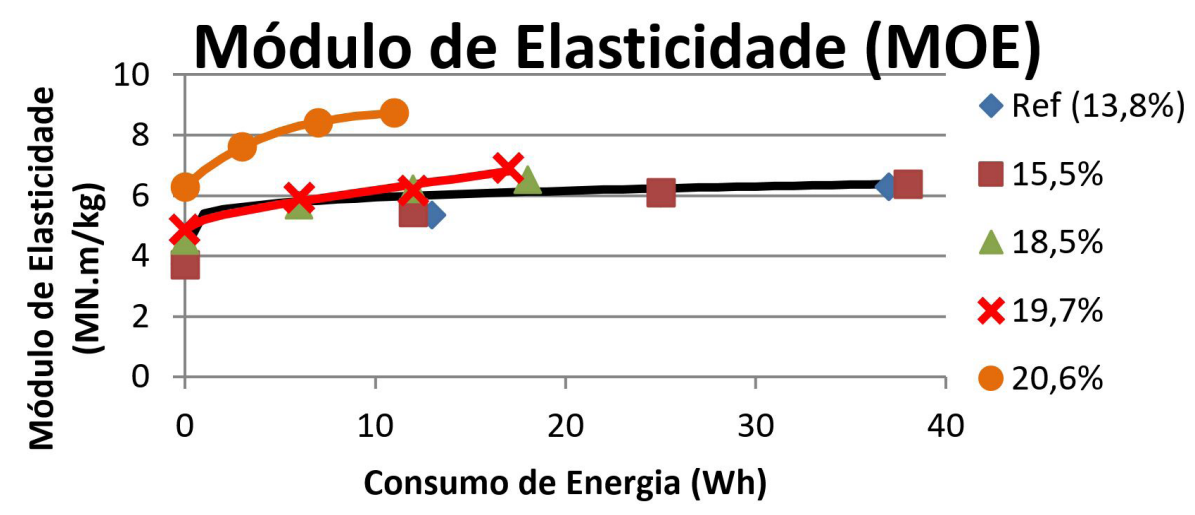

Figura 10. MOE das polpas Referência, $X 5, X 10, X 15$ e X20 em função do consumo de energia após cada nível de refino. Figure 10. MOE of Reference pulps, X5, X10, X15 and X20 in terms of the energy consumption after each level of refining.

curva comum pode ser usada como estimativa para as amostras iguais, enquanto para as demais polpas o modelo estatístico não é o mesmo, existindo assim, diferenças para estes experimentos.

A gramatura e a espessura são também consideradas propriedades importantes na distribuição e no comércio de papéis, principalmente do segmento de papéis de escrita. A associação da gramatura com a espessura da folha resulta no peso específico aparente (PEA) e no volume específico aparente (VEA) do papel. Tem sido relatado (ANJOS et al., 2005, MOLINA et al., 2008) que polpas com alto teor de hemiceluloses tendem a formar papéis com maior densidade (PEA) e menor volume (VEA). A Figura 11 indica que polpas com maior conteúdo de xilanas formam papéis mais densos, enquanto tendências semelhantes no sentido oposto foram observadas para o volume específico aparente do papel. Para os papéis produzidos com as polpas tendo cerca de $20 \%$ de xilanas os valores de VEA foram muito baixos.

Quando uma luz é incidida num conjunto de folhas de polpa, ela é refletida, espalhada e absorvida, sendo esses os fenômenos responsáveis pela propriedade óptica do papel. A Figura 12

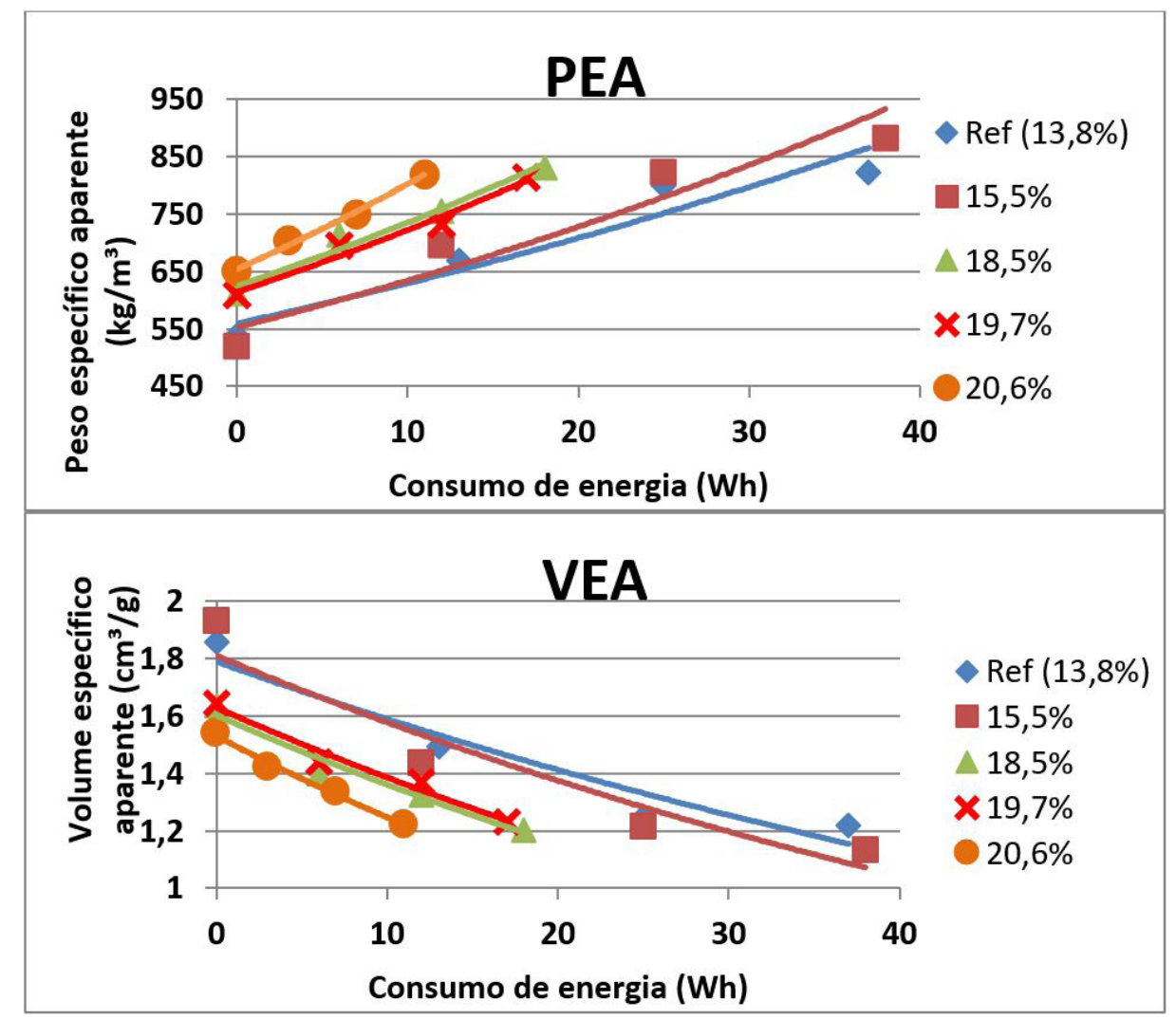

Figura 11. Resultados do peso específico aparente e volume específico aparente das polpas Referência, $X 5, X 10, X 15$ e X20 em função do consumo de energia.

Figure 11. Results of apparent specific weight and apparent specific volume of reference pulps, $X 5, X 10, X 15$ and $\mathrm{X} 20$ in terms of the energy consumption. 
Resende, Colodette e Andrade. - Utilização do filtrado CCE na deslignificação com oxigênio e seus efeitos na branqueabilidade, refinabilidade e

propriedades do papel

apresenta curvas de opacidade e coeficiente de dispersão de luz (CDL) para os papéis produzidos em função do consumo de energia de refino das polpas Referência, X5, X10, X15 e X20. De modo geral, o refino causou a queda na opacidade e CDL de todas as amostras. Quando a polpa foi refinada, as fibras passaram a ficar mais intrelaçadas e unidas, tornando o meio menos heterogêneo por meio da redução da quantidade de ar pela compactação da folha teste. Com isso, a absorção da luz incidida passou a ser menor com a maior facilidade da passagem de luz pelo papel, ocasionando a redução da opacidade da folha e redução do coeficiente de espalhamento de luz no interior da folha (Figura 12).O teor de xilanas da polpa aumenta a interação entre fibras, contribuindo ainda mais para o aumento da compactação e interação entre fibras, de forma que as polpas com maiores teores de xilanas apresentaram menores valores de opacidade e CDL.

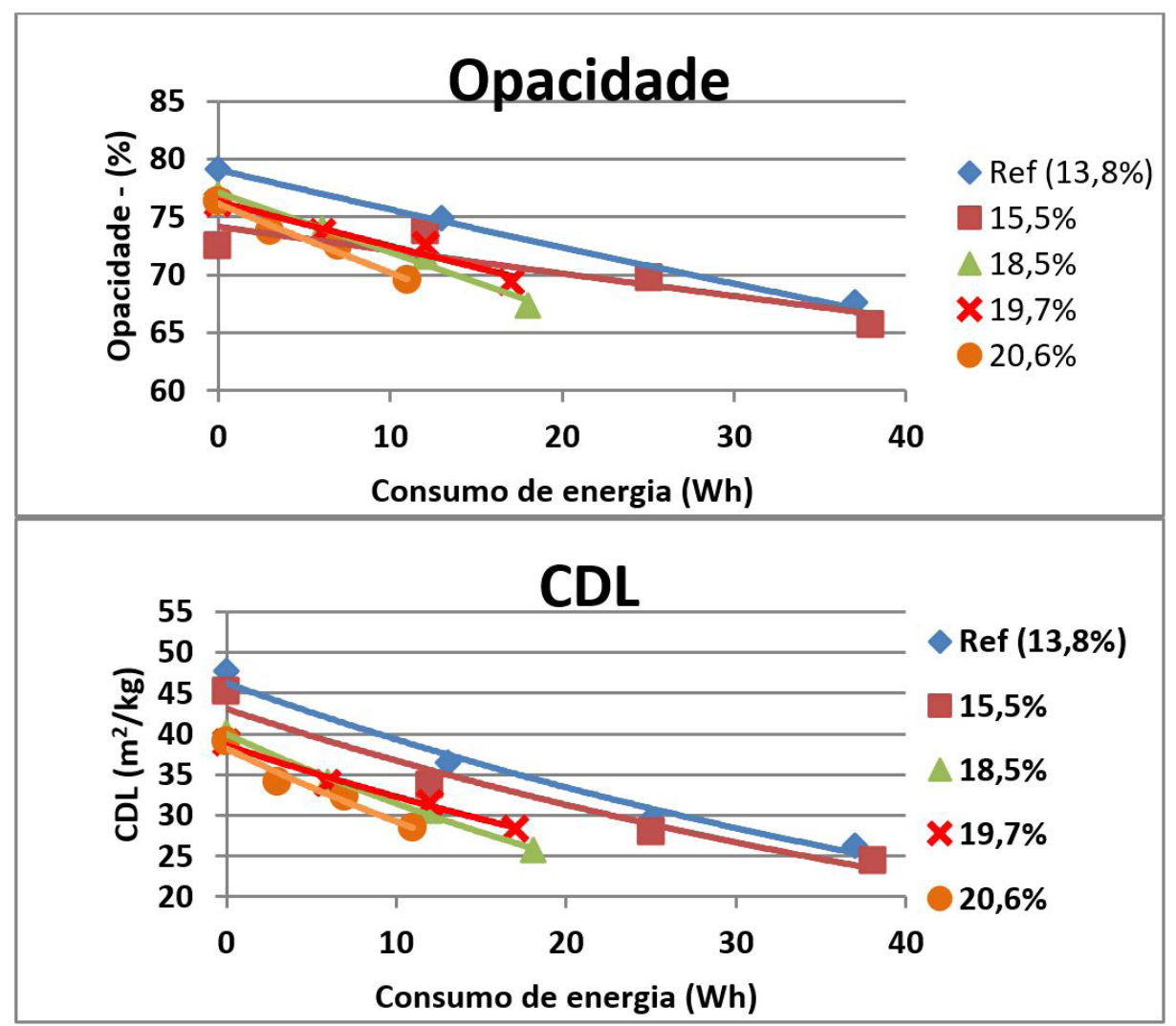

Figura 12. Resultados de opacidade e coeficiente de dispersão de luz (CDL) das polpas Referência, X5, X10, X15 e X20 em função do consumo de energia.

Figure 12. Opacity and scattering coefficient esults of Reference pulps, $X 5, X 10, X 15$ and $X 20$ in terms of the energy consumption.

\section{CONCLUSÕES}

Foi possível modificar a quantidade de xilanas em polpas de eucalipto por meio da redeposição de xilanas, extraídas e precipitadas de licor CCE, na etapa de pré- $\mathrm{O}_{2}$;

Foi possível produzir polpas branqueadas com conteúdos de xilanas variando na faixa de 13,9 a $20,6 \%$;

O rendimento das polpas com adição de xilanas aumentou de $-1,3$ a 4,5\%em relação a polpa referência;

O conteúdo de xilanas afetou negativamente a branqueabilidade das polpas, de modo que o aumento de xilanas levou ao uso de maiores dosagens de reagentes, aumentando o custo do branqueamento;

As operações no branqueamento não afetaram o conteúdo de xilanas das polpas;

O consumo de energia de refino foi influenciado pelo conteúdo de xilanas nas polpas, sendo que o maior teor de xilanas facilita o refino das polpas; 
A operação de refino não afetou o conteúdo de xilanas das polpas;

De modo geral, as propriedades de resistência dos papéis branqueados foram beneficiadas pela adição de xilanas;

Para níveis muito altos de refino ocorreram quedas de resistência ao rasgo para os papéis produzidos, principalmente com maior teor de xilanas;

Os papéis branqueados com elevado conteúdo de xilanas apresentaram resistências superiores, mostrando-se muito atrativos para o segmento de imprimir e escrever.

\section{REFERÊNCIAS BIBLIOGRÁFICAS}

ANJOS, O.; SANTOS, A.; SIMÕES, R. Efeito do teor de hemiceluloses na qualidade do papel produzido com fibra de Eucalipto. In: CONGRESSO FLORESTAL NACIONAL, 5, 2005. Actas das comunicações... Rui Silva e Fernando Páscoa Editores, 2005. Disponível em: < https://core.ac.uk/download/pdf/62720755.pdf >. Acesso em: 20 jan. 2018.

BARBOSA, B. M.; COLODETTE, J. L.; SOARES, M. C. S. M.; GOMES, V. J.; OLIVEIRA, R. C. Effects of xylan in eucalyptus pulp production. Cerne, Lavras, v. 22, n. 2, p. 207-214, 2016.

CASTANHO, C. G.; OLIVEIRA, R. C. Estudos de aproveitamento de rejeito fibroso industrial da polpação kraft de eucalipto na produção de papéis reciclados. In: CONGRESSO ANUAL DE CELULOSE E PAPEL DA ABTCP, 33., 2000. São Paulo. Anais... São Paulo: ABTCP, 2000.

DANIELSSON, S. Xylan reactions in kraft cooking - Process and product considerations. Tese. (Doutorado em Química) Royal Institute of Technology, Sweden, 2007.

MILANEZ, A. et al. Influencia das hemiceluloses nas propriedades óticas e físico-mecanicas da polpa. In: CONGRESSO ANUAL DA ABCP: SEMANA DO PAPEL. 15., 1982, São Paulo Anais... ABTCP, 1982. p. 22-26.

MOKFIENSKI, A; COLODETTE, J. L.; GOMIDE, J. L.; CARVALHO, A. M. M. L. A importância relativa da densidade da madeira e do teor de carboidratos no rendimento de polpa e na qualidade do produto. Ciência Florestal, Santa Maria, v. 18, n. 3, p. 401-413, 2008.

MOLINA, E.M. A.; MOGOLLÓN, G.; COLODETTE, J.L. Efecto de las xilanas en la refinabilidad y propiedades físico-mecánicas de pulpa kraft de eucalyptusspp. In: CONGRESO IBEROAMERICANO DE INVESTIGACIÓN EN CELULOSA Y PAPEL. 2008., Guadalajara. Anais.. Guadalajara: Riadicyp, 2008

PEDRAZZI, C. Influência das xilanas na produção e nas propriedades de polpas de eucalipto para papéis. 2009. 195 p. Tese (Doutorado em Ciência Florestal) - Universidade Federal de Viçosa, Viçosa, 2009.

REGAZZI, A. Teste para identificar a identidade de modelos de regressão e igualdade de alguns parâmetros num modelo polinomial ortogonal. Revista Ceres. Viçosa, v. 40, n. 228, p. 176-195, 1993.

SALOMÃO, K. G. Características e branqueabilidade de polpas kraft/ polissulfeto de eucalyptus e de pinus. 2001. Dissertação (Mestrado em Ciência Florestal) - Universidade Federal de Viçosa, Viçosa, 2001.

SCHÖNBERG, C.; OKSANEM, T.; SUURNÄKKI, A.; KETTUNEM, H.; BUCGHERT, J. The importance of xylan for the strength properties of spruce kraft fibres. Holzforschung, Berlin, v. 55, p. 639-644, 2001.

SOARES, M. C. S. M., Métodos alternativos para deposição de xilanas em polpas de eucalipto. 2009. 56 p. Dissertação (Mestrado em Qualidade da Madeira, Tecnologia de Celulose e Papel) - Universidade Federal de Viçosa, Viçosa, 2009.

SOARES, M. C. M.; COLODETTE, J. L.; PEDRAZZI, C. Xylans deposition onto eucalyptus pulp fi bers during oxygen delignifi cation: part 1 , the infl uence of $\mathrm{NaOH}$ charge, reaction time and temperature. Lisbon: CIADICYP, 2010.

SIXTA, H. Pulp purification. In: Handbook of pulp. Weinheim: Wiley-VCH, 2006. v. 2. p. 933-965.

YLLNER, S.; ENSTRÖM, B. Studies of the adsorption of xylan on cellulose fibres during the sulphate cook. Part. 2. Svensk Papperstidning, v. 60, n. 6, p. 449, 1957.

Recebido em: 01/12/2017

Aceito em: 05/06/2018

Sci. For., Piracicaba, v. 46, n. 120, p. 657-669, dez. 2018

DOI: dx.doi.org/10.18671/scifor.v46n120.14 